\title{
Rapid diagnosis of vaginal candidosis by latex particle agglutination
}

\author{
VALERIE HOPWOOD, ${ }^{*} \ddagger$ EGV EVANS, ${ }^{*}$ JA CARNEY $\dagger$ \\ From the *Department of Microbiology, University of Leeds and Regional Mycology Laboratory, General \\ Infirmary, Leeds and the †Research and Development Unit, Mercia-Brocades Diagnostics Ltd, Braintree, \\ Essex
}

SUMMARY Vaginal swabs from women who on clinical evidence were thought to have vaginal candidosis were examined for yeasts by conventional laboratory methods (microscopy and culture) and also assayed for Candida antigens using a rapid ( $3 \mathrm{~min})$ slide latex particle agglutination test. Results showed that a diagnosis of vaginal candidosis based on clinical criteria alone is unreliable: only half of the women were subsequently confirmed as having candidosis by microscopy and culture. The new slide latex particle agglutination test gave better results, with $100 \%$ specificity, $80 \%$ sensitivity, high predictive values $(\geqslant 91 \%)$, and an overall diagnostic efficiency of 93\%.

From the results of this preliminary study, slide latex particle agglutination looks a promising, rapid alternative to conventional laboratory methods for confirming a clinical diagnosis of vaginal candidosis and has the considerable advantage that it can be conveniently used in a clinical setting.

Candida vaginitis (thrush) is one of the most frequently encountered forms of superficial candidosis. It usually arises due to alterations in the normal physiological state of the host and is a common infection in, for example, poorly controlled diabetics, ${ }^{12}$ women taking oral contraceptives,,$^{3-5}$ and in those in the third trimester of pregnancy. ${ }^{67}$ Vaginal candidosis presents mainly in general practice, but it is also frequently encountered in family planning; obstetrics, gynaecology, and venereology clinics.

A presumptive diagnosis of Candida vaginitis may be based on clinical features such as a pruritis, milky white discharge, erythema and oedema, and the presence of white, curd like patches on the epithelial surface of the vulva, vagina, and cervix. Some of these features, however, are also seen in other forms of vaginitis, particularly in infection due to Trichomonas or gonorrhoea, and therefore an unequivocal diagnosis of candidosis also requires the demonstration of yeasts in material from lesions by both microscopy and culture.$^{8}$ In practice, the diag-

‡Present address: Department of Microbiology, Bristol Royal Infirmary, Bristol, BS2 8HW.

Accepted for publication 21 December 1984 nosis of most cases of vaginal candidosis is made on purely clinical grounds because it is often impracticable or too time consuming to perform the necessary confirmatory tests in a clinical environment and laboratory facilities are not readily available. Moreover, if material is sent to the laboratory there is an inevitable delay before results are available. This less than satisfactory situation means that many women with vaginitis continue to suffer discomfort because of a delay in initiating appropriate treatment and, furthermore, it is costly in terms of the inappropriate medicaments given to some patients.

We have investigated the possibility of rapid ( 3 min) diagnosis of vaginal candidosis using a slide latex particle agglutination (SLA) test for the detection of Candida antigens in vaginal secretions and have compared its efficacy with clinical and conventional laboratory diagnosis.

\section{Material and methods}

An SLA reagent for the detection of Candida cell wall antigens was developed and evaluated for the rapid diagnosis of vaginal candidosis. Vaginal swabs from women considered clinically to have Candida vaginitis were first examined for yeasts by microscopy and culture and then tested blind with the 
SLA reagent. Results were compared.

\section{CLINICAL SPECIMENS}

A total of 87 vaginal swabs received by the Regional Mycology Laboratory at the General Infirmary, Leeds, over a period of four months were included in the study. All the swabs had been taken by general practitioners from women considered clinically to have vaginal candidosis and who were taking part in a multicentre trial of a new antifungal treatment. The swabs were sent in transport medium (Sterilin Ltd, Teddington, England) to Leeds for confirmatory laboratory diagnosis. Thirty six swabs were taken from patients before the start of antifungal treatment and the remainder (51) were follow up swabs to assess the success of treatment.

\section{LABORATORY DIAGNOSIS}

Microscopy and culture

Material from the swabs was examined microscopically in potassium hydroxide $(10 \% \mathrm{wt} / \mathrm{vol})$ for the presence of yeast cells and mycelium or pseudomycelium. Material was also cultured on Sabouraud's dextrose agar containing chloramphenicol $(0.05 \mathrm{~g} / \mathrm{l})$ in petri dishes at $37^{\circ} \mathrm{C}$ and cultures examined at 24 and $48 \mathrm{~h}$. Any yeasts that developed were identified using the germ tube test ${ }^{9}$ and the API 20C Aux yeast identification system (API Lab Products, Basingstoke, England).

Women were considered to have Candida infection when swabs were positive for yeasts by both microscopy and culture. When microscopy was negative and only small numbers of yeasts $(<10$ colonies) were recovered in culture, this was taken to indicate colonisation with Candida rather than infection.

\section{SLA test}

1 Preparation of reagents. An antiserum was raised in New Zealand White rabbits using a partially purified cell wall fraction ${ }^{10}$ of $C$ albicans 3153 , serotype A (ATCC No 28367) as the immunogen. Purified immunoglobulins were obtained from this antiserum by caprylic acid precipitation," followed by diethylaminoethyl Sephadex chromatography. Immunoglobulins were lyophilised and stored under vacuum in sealed ampoules.

Extensive preliminary work was performed to determine the optimum concentration of immunoglobulins required to sensitise the latex particles so as to give maximum sensitivity compatible with stability.

Polystyrene latex particles $0.8 \mu \mathrm{m}$ diameter (Merrel-Dow Ltd, Hounslow, England) were adjusted to a $1 \%(\mathrm{vol} / \mathrm{vol})$ suspension in distilled water. Ten millilitres of immunoglobulin, at a con- centration of $100 \mu \mathrm{g} / \mathrm{ml}$ in glycine buffered saline, $\mathrm{pH} 8.2$ (GBS), was added to $10 \mathrm{ml}$ of latex suspension and the two were mixed vigorously for 2-3 $\mathrm{min}$. Ten millilitres of GBS containing $1 \%$ bovine serum albumin (GBS-BSA) was added and mixed thoroughly. The latex reagent was sonicated (12 $\mu \mathrm{m}$ amplitude) for $6 \mathrm{~s}$ to break up any aggregates and centrifuged $(7000 \mathrm{~g}$ for $10 \mathrm{~min})$. After washing twice with GBS-BSA $(30 \mathrm{ml})$ the latex particles were resuspended in $10 \mathrm{ml}$ of GBS-BSA and stored at $4^{\circ} \mathrm{C}$.

The sensitivity of the latex reagent for antigen detection was determined by reacting against $C$ albicans cell wall mannan ${ }^{12}$ diluted in GBS, and its reactivity was checked every month to ensure that it had not deteriorated. The reagent used for the study could detect $500 \mathrm{ng} / \mathrm{ml}$ mannan in GBS.

2 Test procedure. Swabs were agitated in $100 \mu \mathrm{l}$ of GBS for 1-2 min and the washings centrifuged ( $1500 \mathrm{~g}$ for $5 \mathrm{~min}$ ) to remove particles of transport medium. Twenty five microlitres of sensitised latex reagent were added to $25 \mu$ l of supernatant on a black glass slide and the two reagents were mixed thoroughly with an applicator stick. After manual rotation of the slide for $3 \mathrm{~min}$, agglutination was recorded macroscopically on a scale of negative to 4+ (coarse agglutination, background clear). Swabs giving an agglutination score of $1+$ (fine granular agglutination, background milky) or greater were recorded as positive.

\section{Results}

\section{CLINICAL AND LABORATORY DIAGNOSIS}

Of the 36 vaginal swabs taken from women with clinically suspected vaginal candidosis before treatment, $18(50 \%)$ were subsequently confirmed as

Evaluation of slide latex particle agglutination (SLA) test for the diagnosis of vaginal candidosis. Comparison of the number of patients with suspected infection who were positive by microscopy and culture and by SLA.

\begin{tabular}{llll}
\hline & \multicolumn{2}{l}{ Latex test } & \\
\cline { 2 - 4 } & Positive & Negative & Total \\
\hline $\begin{array}{l}\text { Vaginal candidosis* } \\
\text { Positive }\end{array}$ & 24 (a) & 6 (b) & 30 (c) \\
Negative & 0 (d) & $57(\mathrm{e})$ & 57 (f) \\
Total & 24 (g) & 63 (h) & 87 (i) \\
\hline
\end{tabular}

*Vaginal candidosis positive $=$ microscopy and culture positive.

Vaginal candidosis negative $=$ microscopy positive and culture negative; microscopy negative and $<10$ yeast colonies on culture.

Sensitivity $(\mathrm{a} / \mathrm{c})=80 \%$; specificity $(\mathrm{e} / \mathrm{f})=100 \%$; predictive value positive $(\mathrm{a} / \mathrm{g})=100 \% ;$ predictive value negative $(\mathrm{e} / \mathrm{h})=91 \% ; \sigma$ efticiency $\left(\frac{a+e}{i}\right)=93 \%$ 
cases of Candida infection by conventional laboratory investigations (microscopy and culture). Most of the infections (15) were caused by Calbicans, although in two instances $C$ parapsilosis was responsible and in one case $C$ glabrata.

\section{SLA TEST}

The results obtained with the latex reagent are summarised in the Table. Of the 87 vaginal swabs tested, $30(34 \%)$ were positive by microscopy and culture, and of these $24(80 \%)$ were also positive with the SLA reagent. False negative results were obtained in six $(20 \%)$ instances, but no false positive results were obtained.

\section{SLA PERFORMANCE}

The latex reagent had an excellent specificity $(100 \%)$ and consequently a predictive value positive of $100 \%$. The sensitivity was $80 \%$, giving a predictive value negative of $91 \%$ and an overall efficiency of $93 \%$.

\section{Discussion}

Candida could not be recovered from half of the women who were clinically thought to have vaginal candidosis. It is unlikely that the failure to detect yeasts in swabs from these women can be attributed to the poor sensitivity of the tests employed; in most of those women found to be positive by the laboratory, numerous yeast cells and mycelium could be seen on direct microscopy and large quantities of Candida were recovered in culture without any difficulty. Rather, the results confirm the difficulty of diagnosing vaginal candidosis on purely clinical grounds and the desirability of carrying out confirmatory laboratory tests. The lack of facilities for such tests, the expertise required, and the time delay before results are available make microscopy and culture less than satisfactory. Clearly, however, any new test for the diagnosis of these infections would need to be rapid and easy to perform to confer any advantage over these conventional laboratory procedures. An SLA test with the required sensitivity and specificity would fit these requirements.

Latex particle agglutination tests were first described for the diagnosis of rheumatoid arthritis. ${ }^{13}$ Since then, the production of new latex reagents has enabled the development of rapid and more sensitive agglutination assays, which have gained wide application for the diagnosis of bacterial infections. ${ }^{14-16}$ In mycology, a latex test for the detection of antigen has been used routinely for the diagnosis of cryptococcosis since $1963,{ }^{17}$ and more recently an SLA test has been described for the detection of antigenaemia in patients with systemic candidosis. ${ }^{18}$

The SLA reagent evaluated in this study had total specificity with high predictive values, and even though there is scope for improving its sensitivity $(80 \%)$, it was still considerably better for the diagnosis of vaginal candidosis than reliance on clinical features alone.

The false negative latex results seen in this survey did not appear to be related to the quantity of Candida grown from the swabs since most swabs gave profuse (confluent) growth of yeast on culture, and, conversely, swabs giving fewer colonies on culture scored up to $3+$ by SLA. There are a number of other possible explanations. False negative reactions could, for example, be obtained in infections caused by Candida species other than $C$ albicans. In this survey one of the false negatives in fact occurred with the $C$ glabrata infection, although the two $C$ parapsilosis infections both gave positive results in the SLA test. Furthermore, it is possible that some false negative reactions were obtained with infections caused by isolates of the less frequently encountered $C$ albicans serotype B. The antibodies used to sensitise the latex particles in this study were raised against $C$ albicans serotype $A$ and differences in cross reactivities between the cell wall antigens of the two serotypes, as well as between different Candida species, have been shown. ${ }^{14}$ It is most likely, however, that the majority of the false negative reactions in this survey resulted from the removal of a substantial proportion of the material from the swabs for microscopy and culture before testing with the SLA reagent. The priority given to conventional laboratory methods was dictated by the fact that swabs were primarily collected for assessment of patients in an antifungal trial. Swabs taken from patients and tested directly by SLA would not suffer this disadvantage and, moreover, would not require the centrifugation step used in this survey to remove particles of transport medium. The use of latex particles coated with antibodies to a wider range of Candida species/serotypes may nevertheless contribute to an improved sensitivity and this aspect is under investigation. Even as it stands, the test gives acceptable results and is worthy of further evaluation.

In conclusion, this preliminary study shows the feasibility and potential of SLA for the rapid confirmatory diagnosis of vaginal and possibly other forms of superficial candidosis. The test can easily be carried out in a clinical setting and so offers considerable benefits to doctor and patient.

We thank Miss J Taylor and Mrs F Day for their help with development of the SLA test and Mr RA Forster for his help, especially in obtaining clinical 
specimens. We are grateful to the Science Research Council and Mercia-Brocades Diagnostics Ltd for financial support and for providing one of us ( $\mathrm{VH})$ with a CASE research studentship.

\section{References}

' Sonck CE, Somersalo $O$. The yeast flora of the anogenital region in diabetic girls. Arch Dermatol 1963;88:846-52.

${ }^{2}$ Nagesha CN, Anathakrishna NC. Clinical and laboratory study of monilial vaginitis. Am J Obstet Gynecol 1970;107:1267-8.

${ }^{3}$ Porter PS, Lyle JS. Yeast vulvovaginitis due to oral contraceptives. Arch Dermatol 1966;93:402-3.

${ }^{4}$ Diddle AW, Gardner WH, Williamson PJ, O' Connor KA. Oral contraceptive medications and vulvovaginal candidosis. Obstet Gynecol 1969;34:373-7.

${ }^{5}$ Catterall RD. Influence of gestogenic contraceptive pills on vaginal candidosis. Br J Vener Dis 1971;47:45-7.

- Buck AA, Hasenclever HF. Epidemiologic studies of skin reactions and serum agglutinins to Candida albicans in pregnant women. Am J Hyg 1963;78:232-40.

${ }^{7}$ Pedersen GT. Yeasts isolated from the throat, rectum and vagina in 60 women examined during pregnancy and $1 / 2$ to 1 year after labour. Acta Obstet Gynecol Scand 1964;42(suppl 6):47-51.

${ }^{8}$ Odds FC. Candida and candidosis. Leicester: Leicester University Press, 1979.

' Taschdjian CL, Burchall JJ, Kozinn PJ. Rapid identification of Candida albicans by filamentation on serum and serum substitutes. Am J Dis Child 1960;99:212-5.

${ }^{10}$ Reiss E, Stone SH, Hasenclever HF. Serological and cellular immune activity of peptidoglucomannan fractions of Candida albicans cell walls. Infect Immun 1974;9:881-90.

"Steinbuck M, Audran R. The isolation of IgG from mammalian sera with the aid of caprylic acid. Arch Biochem Biophys $1969 ; 134: 279-84$.

${ }^{12}$ Peat S, Whelan WJ, Edwards TE. Polysaccharide of bakers' yeast. Part IV: Mannan. J Chem Soc 1961;1:29-34.

${ }^{13}$ Singer JM, Plotz CM. The latex fixation test, I. Application to the serological diagnosis of rheumatoid arthritis. Am J Med 1956;21:888-92.

${ }^{14}$ Coonrod JD, Rylko-Bauer B. Latex agglutination in the diagnosis of pneumococcal infection. J Clin Microbiol 1976;4:168-74.

is Ward JI, Siber GR, Scheifele DW, Smith DH. Rapid diagnosis of Haemophilus infuenzae type B infections by latex particle agglutination and counterimmunoelectrophoresis. J Pediatr 1978;93:37-42.

${ }^{16}$ Bortolussi R, Worf AJ, Casey S. The latex agglutination test versus counterimmunoelectrophoresis for rapid diagnosis of bacterial meningitis. Can Med Assoc J 1982;127:489-92.

17 Bloomfield N, Gordon MA, Elmendorf Jr DF. Detection of Cryptococcus neoformans antigen in body fluids by latex particle agglutination. Proc Soc Exp Biol Med 1963;114:64-7.

${ }^{18}$ Gentry LO, Wilkinson ID, Lea AS, Price MF. Latex agglutination test for detection of Candida antigen in patients with disseminated disease. Eur J Clin Microbiol 1983;2:122-8.

${ }^{14}$ Hasenclever HF, Mitchell WO. A study of yeast surface antigens by agglutination-inhibition. Sabouraudia 1964;3:288-300.

Requests for reprints to: Dr EGV Evans, Regional Mycology Laboratory, The General Infirmary at Leeds, Great George Street, Leeds LS1 3EX, England. 